\section{Langjährige DGfK-Mitgliedschaft} Januar / Februar 2015

Folgenden Mitgliedern dankt die Deutsche Gesellschaft für Kartographie für ihre langjährige Treue:

\section{Mitglied seit 60 Jahren:}

Dipl.-Ing.(FH) Heribert Röttgermann, 21465 Reinbek

ADAC e.V., 81373 München

\section{Mitglied seit 50 Jahren:}

Dipl.-Ing.(FH) Kurt Kirchhöfer, 60439 Frankfurt am Main

\section{Mitglied seit 40 Jahren:}

Dipl.-Ing.(FH) Reinhold Knödel, 70619 Stuttgart

Dipl.-Ing. Jürgen Lamping, 93077 Bad Abbach

Prof. Dipl.-Math. Hans F. Kern, 69122 Heidelberg

Dr.-Ing. Roland Schittenhelm, 14167 Berlin

Dipl.-Geogr. Wolfgang Meißner, 44287 Dortmund

Marianne Schultze, 53347 Alfter Fritz Kelnhofer, 1140 Wien,Österreich

\section{- Mitglied seit 25 Jahren:}

Dipl.-Ing. Karl Meier, 77815 Bühl Dipl.-Ing.(FH) Hartmut Lehmann, 12347 Berlin

Dipl.-Ing. Cordula Mann, 35037

Marburg

Dr.-Ing. Jörn Sievers, 63263 Neu-

Isenburg

Dr. rer.nat. Günter Friedlein, 04289

Leipzig

Dipl.-Ing.(FH) Bernhard Spachmüller, 91126 Schwabach

Dr.-Ing. Andreas Illert, 63303 Dreieich Volker Cebulla, 16540 Hohen Neuendorf

Dipl.-Ing. Reinhilde Weber, 53340

Meckenheim

Dr.-Ing. Bernd Wegener, 31141

Hildesheim

Dipl.-Ing. Heinz Brüggemann, 53424

Remagen

Dipl.-Ing.(FH) Kerstin Nier, 84453

Mühldorf

Dipl.-Geogr. Ralf Bitter, 22147

Hamburg
Dipl.-Ing.(FH) Sylke Dyszack, 30519

Hannover

Brigadier a. D.

Dr. Gerhard Fasching, Wien

\section{Geburtstage}

Januar / Februar 2015

10.1. Dipl.-Ing. Kerstin Dierker, Unterwaldenstr. 19, 16341 Panketal, 60 Jahre

12.1. Dipl.-Phys. Dr. Wolfgang Höppner, Str. N.D. Cocea 9, 550370 Sibiu, Rumänien, 70 Jahre

15.1. Dipl.-Ing.(FH) Hans-Eckhard

Schlichting, Lehárstr. 61, 22145

Hamburg, 80 Jahre

19.1. Fritz Kelnhofer,

Forstmeistergasse 19, 1140 Wien, Österreich, 75 Jahre

20.1. Dipl.-Ing. Hans-Oswald Kessler, Pelikanstr. 61a, 70378 Stuttgart, 85 Jahre

22.1. Prof. Dipl.-Geogr. Hans-Jürgen Zylka, Kirchplatz 2 a, 76337 Waldbronn, 75 Jahre

27.1. Dipl.-Ing. Bettina Kuster, Grünstadter Weg 61, 12559 Berlin, 65 Jahre

28.1. Dipl.-Ing. Christine Rülke, Altwölfnitz 4, 01169 Dresden, 65 Jahre 3.2. Dipl.-Ing. Christel Kutzmann, Weinbergstr. 11, 06179 Teutschenthal/ OT Holleben, 75 Jahre

4.2. Dipl.-Ing. Otto von Lom,

Gotenstr. 2, 53424 Remagen, 65 Jahre

6.2. Dipl.-Ing. Ursula Eichhorst, Schneiderstr. 56, 47798 Krefeld, 60 Jahre

18.2. Dr.-Ing. Konrad Großer, Klebendorfer Str. 11, 04425 Taucha, 65 Jahre

19.2. Dipl.-Ing.(FH) Kerstin Fischer, Spreewalder Str. 22, 01239 Dresden, 60 Jahre

20.2. Dipl.-Ing.(FH) Agnes Kamhuber, Ulrich-Nanshaimer-Str. 18, 85652 Pliening, 60 Jahre

27.2. Dipl.-Ing. Manfred Battré, Guerickestr. 5, 30655 Hannover, 90 Jahre

28.2. Peter Uwe Thomsen, Durchschnitt 19, 20146 Hamburg, 75 Jahre

\section{Veranstaltungskalender}

\section{5}

15. Januar 2015, Berlin

nim news - infographics - maps

Infografiker, Redakteure, Journalisten, Kartographen

http://news-infographics-maps.net

4. bis 6. März 2015, Bogotá, Kolumbien

International Interdisciplinary Conference on

Environmental and Geo-Information and Communication

CODATA-Germany, the German National Committee for the ICSU

Committee on Data for Science and Technology

http://CEGeolC2015.net

11. bis 13. Mai 2015, Königslutter

Kartographie: Wirklichkeit - Wahrnehmung - Wissen

Symposium Königslutter der DGfK-Kommission Angewandte

Kartographie - Geovisualisierung

www.angewandte-kartographie.de

11. bis 15. Mai 2015, Berlin

36. International Symposium on Remote Sensing of Environment

German Aerospace Center DLR in partnership with the International Center for Remote Sensing of Environment

http://www.isrse36.org/

27. bis 29. Mai 2015, Korfu, Griechenland

$10^{\text {th }}$ Jubilee Workshop on Digital Approaches to

Cartographic Heritage

ICA Commission on Digital Technologies in Cartographic Heritage xeee.web.auth.gr/ICA-He.....5.htm

18. bis 19. Juni 2015, Potsdam

11. GIS-Ausbildungstagung

GeoForschungsZentrum GFZ Potsdam

http://gis.gfz-potsdam.de/

23. bis 28. August 2015, Rio de Janeiro, Brasilien

$27^{\text {th }}$ International Cartographic Conference

International Cartographic Association (ICA)

http://www.icc2015.org/

15. bis 17. September 2015, Stuttgart

Intergeo und 63. Deutscher Kartographentag

DVW Gesellschaft für Geodäsie, Geoinformation und Landmanagement e. V. Deutsche Gesellschaft für Kartographie

http://www.de.roombites.com/intergeo-2015_deutschland

28. bis 30. Oktober 2015, Berlin

Digital Cultural Heritage

CODATA-Germany, DGfK, ICA u. a.

http://dch2015.net/

\section{6}

14. bis 16. Juni 2016, Potsdam

64. Deutscher Kartographentag

DGfK

Internetadresse wird noch angelegt.

11. bis 13. Oktober 2016, Hamburg

Intergeo 2016

DVW Gesellschaft für Geodäsie, Geoinformation und Landmanagement e. V. Internetadresse wird noch angelegt.

\section{7}

2. bis 7. Juli 2017, Washington, USA

28 $^{\text {th }}$ International Cartographic Conference

International Cartographic Association (ICA)

http://www.icc2017.org/

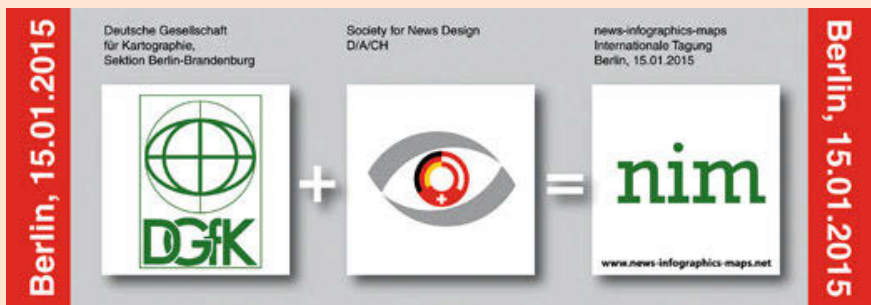

